\title{
Sangrado de tubo digestivo en la pandemia por COVID-19
}

\author{
Ma. Fernanda Torres-Ruiz* \\ Centro de Investigación en Enfermedades Infecciosas, Instituto Nacional de Enfermedades Respiratorias, Ciudad de México, México
}

\begin{abstract}
Resumen
Se realiza una selección de los trabajos presentados en la Digestive Disease Week 2021 (DDW) y en la United European Gastrointestinal Week 2020 (UEGW), en relación con el tema Hemorragia y COVID-19, desde dos enfoques distintos: el impacto de la pandemia en la atención de los pacientes que acudían (o dejaban de acudir) a urgencias con sangrado de tubo digestivo; y, el comportamiento del SARS-CoV-2 como factor de riesgo para presentar hemorragia del tracto gastrointestinal en los pacientes hospitalizados.
\end{abstract}

Palabras clave: COVID-19. Sangrado de tubo digestivo. SARS-CoV-2.

\section{Introducción}

El abordaje de esta temática se realiza desde dos aristas distintas. Por una parte, se revisan los trabajos presentados durante la DDW 2021 y la UEGW 2020 que tuvieron como objetivo evaluar el impacto de la pandemia en la atención y manejo de los pacientes que acudían a urgencias por sangrado de tubo digestivo alto (STDA) durante el inicio de esta; y por otra, los que abordaron el riesgo de presentar STDA secundario a COVID-19 y su tratamiento intrahospitalario.

\section{Impacto de la pandemia en la atención de los pacientes con STDA}

La pandemia por COVID-19 puso bajo presión a los sistemas de salud de todo el mundo. La atención de padecimientos no respiratorios en las salas de urgencias de los hospitales tuvo que ser limitada, dificultando a los pacientes el acceso a servicios hospitalarios. Además, un porcentaje significativo de estos prefería permanecer en casa por miedo a contagiarse del virus. En diferentes países se reconoce una disminución en el número de visitas a urgencias y hospitalizaciones por STDA, así como del número de endoscopias realizadas.

En la DDW se presentaron tres trabajos ${ }^{1-3}$ de diferentes grupos estadounidenses, con una metodología similar, en los que información obtenida de bases de datos o expedientes electrónicos de varios meses del 2020 se comparó con la del mismo periodo, pero de años previos (2017-2019).

El estudio más grande ${ }^{1}$, que incluyó 92 hospitales, reportó una prevalencia del $27.84 \%(28,518 / 102,437)$ hospitalizaciones por STDA en el 2020 vs. $34.22 \%$ $(110,327 / 322,405)$ en el 2019 ( $p<0.001)$, con una disminución del $6.4 \%$ durante la pandemia. En el año más reciente, realizaron un $25 \%$ menos esofagogastroduodenoscopias (EGD) en comparación con el 2019 , sin que esto aparentemente repercutiera en la mortalidad.

Reed, et al. ${ }^{2}$ reportaron una proporción mayor de pacientes que requirieron hospitalización durante el 2020 (73.6 vs. $67.8 \% ; p=0.02$ ), así como de ingresos clasificados como severos (19.3 vs. $14.9 \% ; p=0.03$ ).

En Tailandia ${ }^{4}$, al comparar de manera también retrospectiva el número de pacientes ingresados por

\section{Correspondencia:}


STDA en un hospital de abril a junio del 2020 (periodo COVID-19), con los hospitalizados durante los mismos meses del 2019, los resultados fueron 60 vs. 460 respectivamente. Solo al $43 \%$ se le realizó EGD, y únicamente al $6.7 \%$, en las primeras 24 horas.

Los pacientes ingresados durante la pandemia requirieron más transfusiones de concentrados eritrocitarios $(p=0.002)$, mayor estancia intrahospitalaria $(E I H)$ $(p<0.001)$, y en este estudio sí reportaron una mortalidad mayor a 30 días (OR: 3.41; IC 95\%: 0.86-13.54; $\mathrm{p}=0.097$ ) que aquellos atendidos en el 2019.

En Hong Kong ${ }^{5}$, de acuerdo con un trabajo presentado en la UEGW, el número de hospitalizaciones también disminuyó durante la etapa COVID-19 (35.4 vs. $30.9 ; p<0.01)$, pero no encontraron diferencia, al medir el número de transfusiones, la tasa de resangrado ni de mortalidad, en la evolución de aquellos que requirieron internarse.

Entre los factores que influyeron para la disminución del número de endoscopias realizadas durante el 2020, además de la menor afluencia de pacientes a urgencias, se incluyen las políticas hospitalarias que se implementaron para minimizar el contagio del personal de salud por procedimientos generadores de aerosoles. Dado el acceso limitado al equipo personal de protección, y a salas con presión negativa, en muchas ocasiones se optó por preferir manejos conservadores en los casos de STDA.

En el estudio tailandés ${ }^{4}$ ya mencionado se comparó el desenlace de un grupo de pacientes con STDA tratados conservadoramente contra otro que recibió manejo endoscópico antes de la pandemia. La cohorte con manejo médico presentó mayor mortalidad a 30 días (17.6 vs. $6.2 \% ; p=0.024$ ), posiblemente asociado al diagnóstico de base de malignidad; mayor necesidad de transfusiones y EIH más larga.

Un estudio multicéntrico y prospectivo de Reino Unido ${ }^{6}$ evaluó la seguridad y la eficacia de su política de no hospitalizar pacientes con un score de Glasgow-Blatchford (SGB) menor a 3 que acudían a urgencias con STDA durante la pandemia. De 397 pacientes atendidos en tres meses, al $72.5 \%$ se le realizó EGD y el $36.5 \%$ requirió una intervención endoscópica. La media de EIH fue de 7 días, la mortalidad asociada a sangrado fue del $5 \%$ y la tasa de resangrado de $6.3 \%$. Al compararlo con la información prepandemia de tres centros, la cantidad de casos con STDA por semana, fue menor durante la pandemia, pero la media del SGB fue mayor ( 6.5 vs. $8.3 ; p>0.001$ ). No encontraron diferencia en la tasa de mortalidad asociada a sangrado, por lo que sugieren que la medida adoptada en sus hospitales parece segura.

\section{Impacto de la COVID-19 en el riesgo de sangrado de tubo digestivo (STD)}

Son varios los factores descritos que incrementan el riesgo de STD en los pacientes hospitalizados por COVID-19:

- La enfermedad causada por SARS-CoV-2 se presenta con una amplia gama de síntomas extrapulmonares $^{7}$, entre ellos, trombosis venosa, coagulopatía e isquemia (incluyendo del tracto gastrointestinal).

- Las comorbilidades cardiovasculares son comunes en estos pacientes, y como parte de su manejo, son medicados con antiagregantes plaquetarios 0 anticoagulantes.

- La tromboprofilaxis forma parte del manejo sugerido para la enfermedad COVID-19 moderada y severa.

- Las úlceras por estrés en los pacientes graves los predispone a STD.

Para evaluar la relación entre la gravedad de los pacientes con COVID-19 y el desarrollo de complicaciones gastrointestinales, en un estudio retrospectivo ${ }^{8}$ se revisaron 475 expedientes de pacientes hospitalizados con una prueba positiva para COVID-19 en dos hospitales de Boston; 256 correspondían a casos severos, y en el análisis multivariante tuvieron un mayor riesgo de requerir transfusiones ( 35.7 vs. $4 \%$; $p<0.001$ ) y de presentar STD durante la hospitalización (12.8 vs. $1.5 \% ; p<0.001$ ) en comparación con el grupo de pacientes no severos. El sangrado se manejó de manera conservadora.

En relación con el riesgo de resangrado en los pacientes hospitalizados por COVID-19, Attah, et al. ${ }^{9}$ reportan también de forma retrospectiva, en 56 pacientes, una tasa del $41 \%$ a 30 días. No encontraron diferencia entre los pacientes que recibieron tratamiento médico y los que se les realizó endoscopia (39 vs. $42 \% ; p=0.81$ ), ni entre los que reiniciaron anticoagulación después de la endoscopia o después del tratamiento médico ( 41 vs. $28 \%$; $p=0.47$ ). El $27 \%$ de la cohorte presentó trombosis venosa, y la mitad de los casos se diagnosticó después de suspender la terapia de tromboprofilaxis por STD. La mortalidad asociada a sangrado fue del $9 \%$, sin importar el manejo que hubieran recibido para el STD (24\% endoscópico vs. $29 \%$ médico; $p=0.70$ ). Por lo anterior, parecería viable, considerar el reinicio de la anticoagulación en pacientes en los que el STD ha cedido, incluso si la endoscopia no se ha realizado. 
Por último, para estudiar cuál sería la mejor alternativa de tratamiento en estos pacientes, considerando su riesgo de deterioro respiratorio, además de la exposición del personal de salud a procedimientos generadores de aerosoles, Mauro et al. ${ }^{10}$ describen las características y evolución de 23 pacientes italianos que presentaron STD. De ellos, 7 consumían antiagregantes plaquetarios, 18 tenían indicada anticoagulación (35\% profiláctica y $44 \%$ terapéutica) y $65 \%$ tenía dos o más comorbilidades. La manifestación clínica más frecuente del STD fue la melena (52\%) y se observó en promedio a los 4 días de ElH. En casi la mitad de los pacientes (11) se realizó panendoscopia en las primeras 24 horas, y en 7 más de 24 horas después; los hallazgos fueron, en orden de frecuencia: úlcera péptica, gastritis hemorrágica o erosiva, lesión de Mallory Weiss o de Dieulafoy y várices esofágicas. Solo 6 necesitaron tratamiento endoscópico. El resto recibió tratamiento médico. La mortalidad global fue de $21.7 \%$, sin encontrar diferencia en la mortalidad o resangrado entre los pacientes con endoscopia en menos de 24 horas y aquellos con endoscopia después de 24 horas o con tratamiento médico únicamente. EI SGB fue similar entre ambos grupos.

\section{Conclusión}

Los trabajos analizados muestran que en EE.UU., Tailandia, Hong Kong y Reino Unido la cantidad de hospitalizaciones por STDA disminuyó durante la pandemia. A pesar de recibir pacientes más graves, el número de endoscopias realizadas también fue menor; las consecuencias son controvertidas, sin embargo, en la mayoría de los reportes no se reflejó un aumento de resangrado, ni de mortalidad asociada con el sangrado. Intentar mantener los estándares de atención para las enfermedades no COVID-19 ha de ser una prioridad en todos los sistemas de salud del mundo.

Si bien el nivel de evidencia de los trabajos revisados no es fuerte, se sugiere que los pacientes con COVID-19 severo tienen un riesgo mayor de presentar STD, en comparación con los no severos. La causa más común es la úlcera péptica. El manejo conservador y/o la realización de endoscopia después de $24 \mathrm{~h}$ puede considerarse como una alternativa en pacientes con riesgo de complicaciones respiratorias, sin que esto modifique su desenlace. Futuros estudios son necesarios para evaluar el riesgo de sangrado en pacientes con COVID-19 severo y establecer estrategias para minimizarlo.

\section{Financiamiento}

La presente investigación no ha recibido ayudas específicas provenientes de agencias del sector público, sector comercial o entidades sin ánimo de lucro.

\section{Conflicto de intereses}

Los autores declaran no tener conflicto de intereses.

\section{Bibliografía}

1. Goyal H, Perisetti A, Gajendran M, Chandan S, Anastasiou J, Ali A, et al. Su254 Reduced endoscopy utilization for upper gastrointestinal bleeding during Covid-19 pandemic: A multicenter research networks study. Gastroenterology [Internet]. 2021;160(6 Suppl):S656. Disponible en: https:// www.gastrojournal.org/article/S0016-5085(21)02279-4/fulltext

2. Reddy S, Patel B, Baldelli L, Majithia R, Dougherty M, et al. Fr002 Effect of the COVID-19 pandemic on acute gastrointestinal bleeding. Gastroenterology [Internet]. 2021;160(6 Suppl):S185. Disponible en: https://www. gastrojournal.org/article/S0016-5085(21)01188-4/fulltext

3. Radcliffe MJ, Baig K, Lamboru T, Piech G, Jurkowski Z, Nadpara N, et al. Fr013 COVID-19 Pandemic lowered rates of inpatient gastroenterology consults and endoscopic procedures but did not changes rates of gastrointestinal bleeding or related interventions. Gastroenterology [Internet]. 2021;160(6 Suppl):S190. Disponible en: https://www.gastrojournal.org/ article/S0016-5085(21)01199-9/fulltext

4. Manomaiwong E, Pausawasdi N, Charatcharoenwitthaya P, Kaosombatwattana U. Sa110 Impact of Covid-19 pandemic on the management and clinical outcomes of patients admitted with upper gastrointestinal bledding. Gastroenterology [Internet]. 2021;160(6 Suppl):S424. Disponible en: https://www.gastrojournal.org/article/S0016-5085(21)01723-6/fulltext

5. Lui TKL. Effects of COVID-19 pandemic on hospitalization for upper gastrointestinal bleeding in Hong Kong: A population-based study. United European Gastroenterol [Internet]. 2020;8(8S):856-7. Disponible en: https://ueg.eu/library/effects-of-covid-19-pandemic-on-hospitalization-for-upper-gastrointestinal-bleeding-in-hong-kong-a-population-based-study/241063

6. Dunne P, Livie V, McGowan A, et al. Sa101 Increasing the low risk threshold for patients with upper gastrointestinal bleeding during the COVID-19 pandemic: A prospective, multicenter, feasibility study. Gastroenterology [Internet]. 2021;160(6 Suppl):S421. Disponible en: https:// www.gastrojournal.org/article/S0016-5085(21)01714-5/fulltext

7. Zheng KI, Feng G, Liu WY, Targher G, Byrne CD, Zheng MH. Extrapulmonary complications of COVID-19: A multisystem disease? J Med Virol. 2021;93(1):323-35.

8. Alvencar S, Talanian M, Beano A, Morrison A, Srivoleti P, Sidhu TK, et al. Fr005 Severe COVID-19 associated with increased risk of GI bledding during hospitalization. Gastroenterology [Internet]. 2021;160(6 Suppl):S186. Disponible en: https://www.gastrojournal.org/article/S00165085(21)01191-4/fulltext

9. Attah E, Smith E, Martin T, Tewni S, Hajifathalian K, Sharaiha RZ, et al. Fr012 The observed risk of recurrent bleeding and thromboembolic disease in COVID-19 patients with gastrointestinal bledding. Gastroenterology [Internet]. 2021;160(6 Suppl):S189-S190. Disponible en: https:// www.gastrojournal.org/article/S0016-5085(21)01198-7/fulltext

10. Mauro A, de Grazia F, Lenti MV, Penagini R, Frego R, Ardizzone S, et al. Upper Gl bleeding in COIVD-19 patients: Characteristics and management in a multicenter experience from Northern Italy. United European Gastroenterol [Internet]. 2020;8(8S):856-7. Disponible en: https://ueg.eu/ library/upper-gi-bleeding-in-covid-19-patients-characteristics-and-management-in-a-multicenter-experience-from-northern-italy/235650 